\title{
Enhancement of Brillouin gain efficiency in multiwavelength L-band BEFL by utilizing bi-directional Brillouin pump amplification.
}

\begin{abstract}
Enhancement of Brillouin gain efficiency in a multiwavelength L-band Brillouin-erbium comb fiber laser is presented. In this laser architecture, bi-directional amplification of the injected Brillouin pump signal within the erbium gain medium, before entering the singlemode fiber was utilized. Owing to this bi-directional pre-amplification of the Brillouin pump power, the requirement of a long single-mode fiber to increase the Brillouin gain efficiency was overcome. The shot length of a single-mode fiber was utilized to achieve a high number of output channels with high peak power. Up to 25 output channels with a constant wavelength separation of $0.089 \mathrm{~nm}$ were achieved at $170 \mathrm{~mW}$ of $1480 \mathrm{~nm}$ pump power and $0.54 \mathrm{~mW}$ of Brillouin pump power in a $0.5 \mathrm{~km}$ short single-mode fiber.
\end{abstract}

